\title{
REGGE-EIKONAL APPROACH AND ITS OFF-SHELL EXTENTION VERSUS EXPERIMENTAL DATA
}

\author{
V. A. PETROV AND $\underline{\text { A. V. PROKUDIN }}$ \\ Institute For High Energy Physics, \\ 142284 Protvino , Russia \\ E-mail: petrov@mx.ihep.su \\ prokudin@th1.ihep.su
}

\begin{abstract}
We develop an off-shell extention of the Regge-eikonal approach which automatically takes into account off-shell unitarity. We argue that exclusive vector-meson production cross-sections measured at HERA can be fairly described with classical universal Regge trajectories. No extra "hard" trajectories of high intercept are needed for that.
\end{abstract}

\section{Introduction}

Unitarity condition

$$
\operatorname{Im} T(s, \vec{b})=|T(s, \vec{b})|^{2}+\eta(s, \vec{b})
$$

where $T(s, \vec{b})$ is the scattering amplitude in impact space representation and $\eta(s, \vec{b})$ stands for the contribution of inelastic channels, may be illustrated by the following picture in the momentum space

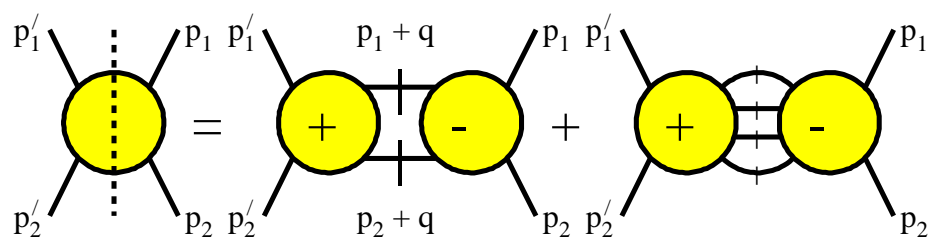

$\operatorname{ImT}\left(p_{1}^{\prime}, p_{2}^{\prime}, p_{1}, p_{2}\right)=\frac{-i}{(2 \pi)^{4}} \int d^{4} q(-2 \pi i)^{2} \delta\left(\left(p_{1}+q\right)^{2}-m^{2}\right) \delta\left(\left(p_{2}-q\right)^{2}-m^{2}\right) \times$ 


$$
\times T^{+}\left(p_{1}+q, p_{2}-q, p_{1}^{\prime}, p_{2}^{\prime}\right) T^{-}\left(p_{1}, p_{2}, p_{1}+q, p_{2}-q\right)+\ldots
$$

where "..." stands for inelastic channels contribution.

It is worth noting that we can include virtual external particles into consideration and in this case the integration in Eq. (11) still goes over particles on mass shell as it was in the case of real external particles, i.e.

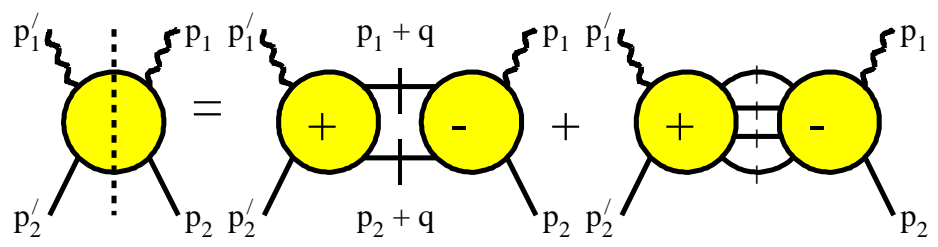

There are many parametrizations that "solve" the s-channel unitarity condition. Examples are $U$-matrix 1 and Eikonal approach 4 .

We choose the eikonal representation as usual

$$
T(s, \vec{b})=\frac{e^{2 i \delta(s, \vec{b})}-1}{2 i},
$$

here $T(s, \vec{b})$ is the scattering amplitude, $\delta(s, \vec{b})$ is the eikonal function. The eikonal function is to be considered as a basic scattering function, which builds up the amplitude. The unitarity condition looks very simple in terms of the eikonal function:

$$
\operatorname{Im} \delta(s, \vec{b}) \geq 0, s>s_{\text {inel }}
$$

In quantum mechanics $\delta(s, \vec{b})$ is related to the (short-range)potential $V$ : 


$$
\left.\delta(s, \vec{b}) \sim \int_{-\infty}^{+\infty} d z V(z, \vec{b}) \sim\right]_{J_{n}=0}^{\pi}
$$

At relativistic energies one can generalize the notion of the potential by introducing a "quasipotential", which is complex and depends on energy 6. As a concrete realizaton of the quasipotential one can take Van-Hove interpretation of the Regge behaviour as a "sum" of all possible one-particle exchanges in the $t$-channel 3 . Combination of the eikonal approach and Regge poles was considered for the first time in 6 . It was shown in 1 in the framework of the asymptotic summation of the perturbative series that the eikonal function $\hat{\delta}(s, t)$ behaves like $s^{1+\Delta}$, where $\Delta>0$.

So one could abstract the idea that there exists some function $\hat{\delta}(s, t)$ with a powerlike growth in $s$ and this is in full conformity with general principles.

We choose the following eikonal function in $t$ space (here $t$ is the transferred momentum)

$$
\hat{\delta}(s, t)=c\left(\frac{s}{s_{0}}\right)^{\alpha(0)} e^{t \frac{\rho^{2}}{4}}
$$

where

$$
\rho^{2}=4 \alpha^{\prime}(0) \ln \frac{s}{s_{0}}+r^{2}
$$

is reffered to as a "reggeon radius".

It means that the eikonal function has a simple pole in $J$ plane and the corresponding Regge trajectory can be written in the following form:

$$
\alpha(t)=\alpha(0)+\alpha^{\prime}(0) t
$$

We make use of linear Regge trajectories in this paper, though there exists some evidence of non-linearity of $\alpha(t)$ when $t$ is high enough.

Functions in $t$ - and $b$-spaces are related by the Fourier-Bessel transformation:

$$
\begin{aligned}
& \hat{f}(t)=4 \pi s \int_{0}^{\infty} d b^{2} J_{0}(b \sqrt{-t}) f(b) \\
& f(b)=\frac{1}{16 \pi s} \int_{-\infty}^{0} d t J_{0}(b \sqrt{-t}) \hat{f}(t)
\end{aligned}
$$

Making use of Eq. (7) we obtain the following $b$-representation of the eikonal function:

$$
\delta(s, b)=\frac{c}{s_{0}}\left(\frac{s}{s_{0}}\right)^{\alpha(0)-1} \frac{e^{-\frac{b^{2}}{\rho^{2}}}}{4 \pi \rho^{2}}
$$


In what follows the term "pomeron" will mean the leading pole of the eikonal function.

For cross sections we use the following normalizations:

$$
\begin{aligned}
& \sigma_{t o t}=\frac{1}{s} \operatorname{Im} T(s, t=0) \\
& \sigma_{e l}=4 \pi \int_{0}^{\infty} d b^{2}|T(s, b)|^{2} \\
& \frac{d \sigma}{d t}=\frac{|T(s, t)|^{2}}{16 \pi s^{2}}
\end{aligned}
$$

\section{Regge-Eikonal Approach}

Let's recall some properties of the Regge-eikonal model.

If $s \rightarrow \infty$, then

$$
\sigma_{\text {tot }} \rightarrow 2 \pi \rho^{2}[c+\ln z-\operatorname{Ei}(-z)]
$$

where $z=\frac{\frac{c}{s_{o}}\left(\frac{s}{s_{o}}\right)^{\Delta}}{2 \pi \rho^{2}}$, here $(\Delta \equiv \alpha(0)-1)$ and $\operatorname{Ei}(z)=\int_{-\infty}^{z} \frac{e^{x}}{x} d x$. At extra high energies we reach the following limit:

$$
\sigma_{t o t} \rightarrow 8 \pi \alpha^{\prime}(0) \Delta\left(\ln \frac{s}{s_{0}}\right)^{2}
$$

i.e. the powerlike behaviour of $\hat{\delta}(s, t)$ leads to the asymptotic behaviour of the full scattering ampitude which functionally saturates the Froissart-Martin bound 6 :

$$
\sigma_{t o t}^{h h} \leq \frac{\pi}{m_{\pi}^{2}}\left(\ln \frac{s}{s_{0}}\right)^{2}, s \rightarrow \infty
$$

If $s \rightarrow \infty$, then

$$
\sigma_{e l} \rightarrow \pi \rho^{2}\left[c+\ln \frac{z}{2}+E i(-2 z)-2 E i(-z)\right]
$$

and thus

$$
\frac{\sigma_{e l}}{\sigma_{t o t}} \rightarrow \frac{1}{2}
$$

\section{The Model}

In our model we choose the following contributions for the eikonal function:

$$
\delta(s, b)=\delta_{\mathbb{P}}^{+}(s, b)+\delta_{\mathbb{O}}^{-}(s, b)+\delta_{f}^{+}(s, b)+\delta_{\omega}^{-}(s, b)
$$


where $\delta_{\mathbb{P}}^{+}(s, b)$ is the pomeron contribution, $\delta_{\mathbb{O}}^{-}(s, b)$ is the odderon ${ }^{\natural}$ contribution, and $\delta_{f}^{+}, \delta_{\omega}^{-}(s, b)$ are contributions of $f$ meson exchange $(C=+1)$ and $\omega$ meson exchange $(C=-1)$.

The trajectories of $f$ and $\omega$ are 0 :

$$
\begin{aligned}
& \alpha_{f}(t)=0.69+0.84 t \\
& \alpha_{\omega}(t)=0.47+0.93 t
\end{aligned}
$$

and

$$
\begin{aligned}
& \delta^{+}(s, b)=\left[i+\operatorname{tg} \frac{\pi(\alpha(0)-1)}{2}\right] \frac{c}{s_{0}}\left(\frac{s}{s_{0}}\right)^{\alpha(0)-1} \frac{e^{-\frac{b^{2}}{\rho^{2}}}}{4 \pi \rho^{2}} \\
& (C=+1) \\
& \delta^{-}(s, b)=\left[i+\operatorname{ctg} \frac{\pi(\alpha(0)-1)}{2}\right] \frac{c}{s_{0}}\left(\frac{s}{s_{0}}\right)^{\alpha(0)-1} \frac{e^{-\frac{b^{2}}{\rho^{2}}}}{4 \pi \rho^{2}} \\
& (C=-1)
\end{aligned}
$$

Where $i+\operatorname{tg} \frac{\pi(\alpha(0)-1)}{2}$ and $i+\operatorname{ctg} \frac{\pi(\alpha(0)-1)}{2}$ are signature coefficients for $C=+1$ and $C=-1$ exchange correspondingly.

\section{$4 \quad$ Fitting procedure}

We explore two cases: with and without odderon contribution.

\subsection{The Model With the Odderon}

The results concerning the trajectories of the pomeron and the odderon are:

$$
\begin{aligned}
& \alpha_{\mathbb{P}}(0)-1=0.11578 \pm 0.003 \quad, \alpha_{\mathbb{P}}^{\prime}(0)=0.27691 \pm 0.00434 \\
& \alpha_{\mathbb{O}}(0)-1=0.11578 \pm 0.00711, \alpha_{\mathbb{O}}^{\prime}(0)=0.27691 \pm 0.00315
\end{aligned}
$$

where $\alpha_{\mathbb{P}}$ and $\alpha_{\mathbb{O}}$ are the intercepts of the pomeron and the odderon, $\alpha_{\mathbb{P}}^{\prime}$ and $\alpha_{\mathbb{O}}^{\prime}$ are the slopes of the pomeron and the odderon respectively. So the fitting procedure lead us practically to the scenario of the weakly degenerate pomeron-odderon trajectory 14. ity 8

$\alpha_{\mathbb{P}}, \alpha_{\mathbb{P}}^{\prime}$ and $\alpha_{\mathbb{O}}, \alpha_{\mathbb{O}}^{\prime}$ were chosen so that the following conditions of unitar-

$$
\begin{aligned}
& \alpha_{\mathbb{P}}(0) \geq \alpha_{\mathbb{O}}(0) \\
& \alpha_{\mathbb{P}}^{\prime}(0) \geq \alpha_{\mathbb{O}}^{\prime}(0)
\end{aligned}
$$

${ }^{a}$ The odderon is the counterpart of the pomeron with $C=-1$ 
would not be violated. The other parameters are in the Table.(11).

Table 1. Parameters of the model with the odderon.

\begin{tabular}{|c|c|c|c|}
\hline$c_{\mathbb{P}}$ & 44.66025 & $c_{\mathbb{O}}$ & 1.92343 \\
$c_{f}$ & 241.69257 & $c_{\omega}$ & 71.20085 \\
$r_{\mathbb{P}}^{2}\left(G e V^{-2}\right)$ & 14.83200 & $r_{\mathbb{D}}^{2}\left(G e V^{-2}\right)$ & 2.18549 \\
$r_{f}^{2}\left(G e V^{-2}\right)$ & 5.55410 & $r_{\omega}^{2}\left(G e V^{-2}\right)$ & 99.99996 \\
\hline
\end{tabular}

We find out that the odderon contribution is significant when $t \neq 0$ and it allows us to qualitatevely describe the differnce in the structure of $\frac{d \sigma}{d t}$ of $\bar{p} p$ and $p p$ 9, 10 while without this contribution we are able to describe only the slopes of $\frac{d \sigma}{d t}$ when $t=0$.

It is worth noting that the discrepancy in the description of $\rho=$ $\frac{R e T}{\operatorname{ImT}}(s, t=0)$ can be understood if we remember that the $\rho$ data are not directly mesaured by experimentalists, but rather extracted from $\frac{d \sigma}{d t}$ experimental data in a model-dependent way.

\subsection{The Model Without the Odderon}

The results concerning the pomeron trajectory is:

$$
\alpha_{\mathbb{P}}(0)-1=0.12169 \pm 0.00286, \alpha_{\mathbb{P}}^{\prime}(0)=0.20933 \pm 0.001379
$$

The other parameters are in the Table.(2).

Table 2. Parameters of the model without the odderon.

\begin{tabular}{|c|c|c|c|}
\hline$c_{\mathbb{P}}$ & 46.599 & & 150.530 \\
$c_{f}$ & 199.630 & $c_{\omega}$ & \\
$r_{\mathbb{P}}^{2}\left(G e V^{-2}\right)$ & 21.204 & & \\
$r_{f}^{2}\left(G e V^{-2}\right)$ & 4.872 & $r_{\omega}^{2}\left(\mathrm{GeV}^{-2}\right)$ & 39.967 \\
\hline
\end{tabular}

The intercept is slightly higher then in case of when the odderon contributes to the eikonal. As is shown, the $t=0$ data can be described without presence of the odderon, while the $t \neq 0$ data can be described, though qualitatively only by means of the odderon. Let's remind our basic assumptions:

- Regge trajectories are linear.

- The $t$ dependence of $\hat{\delta}(s, t)$ is exponential i.e. $\hat{\delta}(s, t) \sim e^{t \rho^{2}}$. 
It is not our aim at present stage to obtain the best fit. What really matters is to fix a set of Regge parameters some of which are considered universal (slopes, intercepts of the trajectories) while others (residues) are not. Universal parameters will be then used for off-shell amplitudes.

\section{Off-Shell Extention Of The Regge-Eikonal Approach}

As it was shown in 10 the amplitude with one off-mass shell particle both in initial and final states (two asteriks)is related to the off-shell eikonals with one and two off-shell particles and on-shell amplitude in the following way

$$
T^{* *}(s, b)=\delta^{* *}(s, b)-\frac{\delta^{*}(s, b) \delta^{*}(s, b)}{\delta(s, b)}+\frac{\delta^{*}(s, b) \delta^{*}(s, b)}{\delta(s, b) \delta(s, b)} T(s, b)
$$

The expansion Eq. 21 can be illustrated by the following figure:

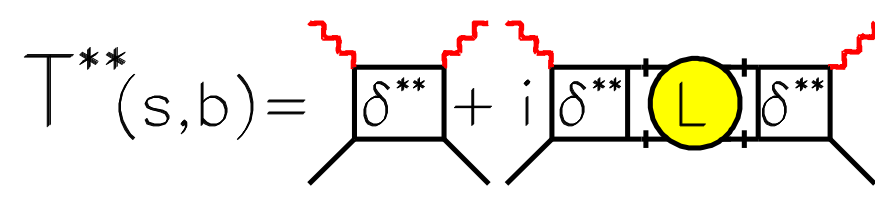

where $L=\sum_{n=2}^{\infty} \frac{(2 i \delta)^{n-2}}{n !}$. The case when only one of the particles is off shell can be considered similarly. One has

$$
T^{*}=\hat{\delta}^{*}+i \hat{\delta}^{*} \circ L \circ \hat{\delta}
$$

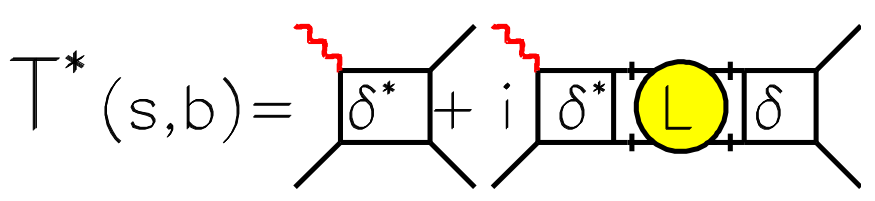

or we rewrite it as follows:

$$
T^{*}(s, b)=\frac{\delta^{*}(s, b)}{\delta(s, b)} T(s, b)
$$


On the basis of Eq.(8) we take the following parametrizations of the off-shell eikonal functions:

$$
\delta_{ \pm}^{*}(s, b)=\xi_{ \pm} \frac{c_{*}\left(Q^{2}\right)}{s_{0}+Q^{2}}\left(\frac{s+Q^{2}}{s_{0}+Q^{2}}\right)^{\alpha(0)-1} \frac{e^{-\frac{b^{2}}{\rho_{*}^{2}}}}{4 \pi \rho_{*}^{2}}
$$

where

$$
\rho_{*}^{2}=4 \alpha^{\prime}(0) \ln \frac{s+Q^{2}}{s_{0}+Q^{2}}+r_{N}^{2}+r_{*}^{2}\left(Q^{2}\right)
$$

and

$$
\delta_{ \pm}^{* *}(s, b)=\xi_{ \pm} \frac{c_{* *}\left(Q^{2}\right)}{s_{0}+Q^{2}}\left(\frac{s+Q^{2}}{s_{0}+Q^{2}}\right)^{\alpha(0)-1} \frac{e^{-\frac{b^{2}}{\rho_{* *}^{2}}}}{4 \pi \rho_{* *}^{2}}
$$

where

$$
\rho_{* *}^{2}=4 \alpha^{\prime}(0) \ln \frac{s+Q^{2}}{s_{0}+Q^{2}}+r_{N}^{2}+r_{* *}^{2}\left(Q^{2}\right)
$$

Coefficients $c_{*}\left(Q^{2}\right), c_{* *}\left(Q^{2}\right)$ are supposed to weakly (not in a powerlike way)depend on $Q^{2}$.

\subsection{Total cross section}

If we consider only the Pomeron exchange (in case of high energies, the main contribution to the scattering amplitude is due to the Pomeron) and set signature coefficient $\xi_{+}$to be equal unity, then we have the following series for the total cross section $\left(\sigma_{\text {tot }}^{* *}=\frac{1}{s} \operatorname{Im} T^{* *}(s, t=0)\right)$ :

$$
\sigma_{\text {tot }}^{* *}=\frac{c_{* *}\left(Q^{2}\right)}{Q^{2}+s_{o}}\left(\frac{1}{x}\right)^{\Delta}-\frac{c_{*}^{2}\left(\frac{1}{x}\right)^{2 \Delta}}{2 \pi\left(Q^{2}+s_{o}\right)^{2} \rho_{*}^{2}} \sum_{n=0}^{\infty} \frac{\left(-\frac{c\left(\frac{s}{s_{o}}\right)^{\Delta}}{2 \pi s_{o} \rho^{2}}\right)^{n}}{(n+2) !} \cdot \frac{\rho^{2}}{2 \rho^{2}+n \rho_{*}^{2}}
$$

here we used the folowing relation $\frac{s+Q^{2}}{s_{0}+Q^{2}} \simeq \frac{s+Q^{2}-m_{N}^{2}}{Q^{2}}=\frac{1}{x}$ (if $s \gg Q^{2}$ and (or) $\left.Q^{2} \gg s_{0}\left(Q^{2} \gg m_{N}^{2}\right)\right)$ Now we can derive the behaviour of the total cross section in different kinematic limits:

- Regge Regime

$$
\sigma_{t o t}^{* *} \rightarrow \frac{\left(s / Q^{2}\right)^{\Delta}}{Q^{2}}\left[c_{* *}-\frac{c_{*}^{2}}{c}\left(\frac{s_{0}}{Q^{2}}\right)^{1+\Delta} \frac{\rho^{2}}{\rho_{*}^{2}}\right]
$$




\section{- Bjorken Regime}

$$
\sigma_{\text {tot }}^{* *} \rightarrow \frac{c_{* *}\left(Q^{2}\right)}{Q^{2}}\left(\frac{1}{x}\right)^{\Delta}-\frac{c_{*}^{2}}{2 c} \cdot \frac{1}{Q^{2}} \cdot\left(\frac{1}{x}\right)^{\Delta} \cdot\left(\frac{s_{o}}{Q^{2}}\right)^{1+\Delta} \cdot \frac{\ln \frac{Q^{2}(1-x)}{s_{o} x}}{\ln \frac{1}{x}}
$$

As we see total cross-section posesses a powerlike behaviour in the Regoge limit, but this is not a violation of unitarity, as the Froissart-Martin bound $\mathbf{6}$ cannot be proven for this case and if we put all particles on the mass shell, then we restore the 'normal' logarithmic asymptotical behaviour $\sigma \sim \ln ^{2} \frac{s}{s_{0}}$. In the Bjorken limit we have strong (powerlike) violation of scaling in the second term.

\subsection{Elastic cross-section}

For the elastic cross section we have the following expression:

$$
\sigma_{e l}^{*}=4 \pi \int_{0}^{\infty} d b^{2}\left|\frac{\delta^{*}}{\delta} T(s, b)\right|^{2}
$$

As far as $q^{\prime 2}=\mu^{2}$, where $\mu$ is the mass of the produced particle, it is natural to set $s_{0}=\mu^{2}$ and now we can derive the following relations:

- Regge Regime

$$
\sigma_{e l}^{*} \rightarrow 16 \pi \alpha^{\prime}(0) \Delta\left(\frac{c_{*}}{c}\right)^{2}\left(\frac{\mu^{2}}{Q^{2}}\right)^{2+2 \Delta}\left(\ln \frac{s}{\mu^{2}}\right)^{2}
$$

- Bjorken Regime

$$
\sigma_{e l}^{*} \rightarrow 8 \pi \alpha^{\prime}(0)\left(\frac{c_{*}}{c}\right)^{2}\left(\frac{\mu^{2}}{Q^{2}}\right)^{2+2 \Delta} \frac{\left(\ln \left(Q^{2} / x\right)\right)^{2}}{\ln (1 / x)}
$$

As we can easily realize

$$
\frac{\sigma_{e l}^{*}}{\sigma_{t o t}^{* *}} \rightarrow 0
$$

when $s \rightarrow \infty$ and $s \gg Q^{2}$ (compare with 14) As to the $Q^{2}$ behaviour, then as far as we have $\Delta=0.116 \pm 0.003$ (see Eq.(18)), we obtain the following dependence:

$$
\sigma_{e l}^{*} \sim \frac{1}{\left(Q^{2}\right)^{2+2 \Delta}}=\left(Q^{2}\right)^{-2.232 \pm 0.006}
$$

This formula is in close agreement with the results of fitting the $Q^{2}$ dependence in $\mathrm{\theta}$, where the power of $Q^{-2}$ is measured as 2.24 . 


\section{Vector meson photoproduction}

For vector meson photoproduction $\gamma^{*} p \rightarrow V p$, where $V=\rho_{o}, \omega, \Phi, J / \Psi, \ldots$

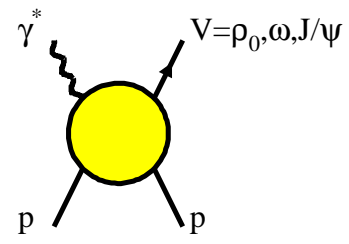

we have the following formula for the amplitude:

$$
T^{*}(s, b)=\frac{\delta^{*}(s, b)}{\delta(s, b)} T(s, b)
$$

here we assume that $T_{\gamma^{*} p \rightarrow V p} \sim T_{V^{*} p \rightarrow V p}$ on the basis of vector dominance 3 . As far as in this process reggeons with $C=-1$ do not contribute, we have the following contributions for the eikonal function:

$$
\delta(s, b)=\delta_{\mathbb{P}}^{+}(s, b)+\delta_{f}^{+}(s, b)
$$

with $\left(s \equiv W^{2}\right)$

$$
\begin{gathered}
\delta_{ \pm}(W, b)=\xi_{ \pm} \frac{c}{W^{2}-\mu^{2}-m_{p}^{2}}\left(\frac{W^{2}-\mu^{2}-m_{p}^{2}}{W_{0}^{2}-\mu^{2}-m_{p}^{2}}\right)^{\alpha(0)} \frac{e^{-\frac{b^{2}}{\rho^{2}}}}{4 \pi \rho^{2}} \\
\rho^{2}(W)=4 \alpha^{\prime}(0) \ln \frac{W^{2}-\mu^{2}-m_{p}^{2}}{W_{0}^{2}-\mu^{2}-m_{p}^{2}}+r_{p}^{2}
\end{gathered}
$$

and

$$
\begin{gathered}
\delta_{ \pm}^{*}(W, b)=\xi_{ \pm} \frac{c_{*}\left(Q^{2}\right)}{W^{2}+Q^{2}-m_{p}^{2}}\left(\frac{W^{2}+Q^{2}-m_{p}^{2}}{W_{0}^{2}+Q^{2}-m_{p}^{2}}\right)^{\alpha(0)} \frac{e^{-\frac{b^{2}}{\rho_{*}^{2}}}}{4 \pi \rho_{*}^{2}} \\
\rho_{*}^{2}(W)=4 \alpha^{\prime}(0) \ln \frac{W^{2}+Q^{2}-m_{p}^{2}}{W_{0}^{2}+Q^{2}-m_{p}^{2}}+r_{p}^{2}+r_{*}^{2}\left(Q^{2}\right)
\end{gathered}
$$

As far as Regge Eikonal approach does not constrain the $Q^{2}$ dependence of residues $c_{*}\left(Q^{2}\right)$ and radii $r_{*}^{2}\left(Q^{2}\right)$ we assume that the main $Q^{2}$ dependence is comprised in the other terms of our formulae and residues slowly depend 
on $Q^{2}$ (they posses logarifmic behaviour)and radii depend on $Q^{2}$ as $\sim \frac{1}{Q^{2}}$. Eventually we have the following parametrizations:

$$
c_{*}\left(Q^{2}\right)=c+c_{1} l^{3}\left(\frac{Q_{o}^{2}+Q^{2}}{Q_{o}^{2}}\right)
$$

and

$$
r_{*}^{2}\left(Q^{2}\right)=r^{2}+\frac{r_{1}^{2}}{\frac{Q_{0}^{2}+Q^{2}}{Q_{0}^{2}}}
$$

where $Q_{0}^{2}=1.0 \mathrm{GeV}^{2}$.

The results of fitting the data to these formulae are in Fig.1.
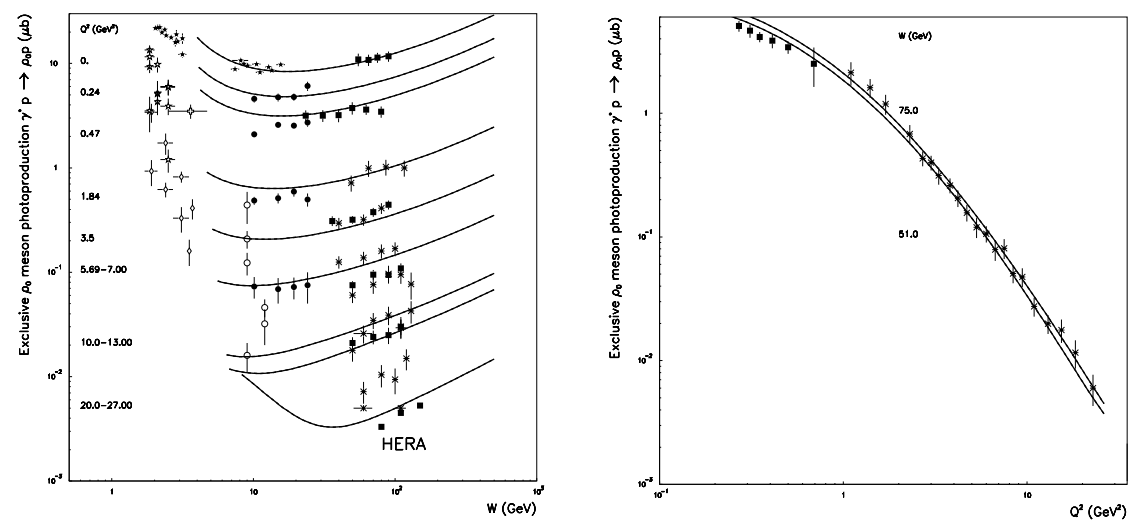

Figure 1. $\rho_{0}$ meson exclusive photoproduction cross-section

As one can see from Fig.2, in the region of energies that are available the asymptotic behaviour of exclusive vector meson photoproduction does not take place and therefore one should take into concideration both Pomeron and $f$ meson exchanges.

For fitting we used the same intercept and slope of the pomeron that have been obtained in the Section 4.1, so that the pomeron in our model is unique and the same for all processes where it takes part. 

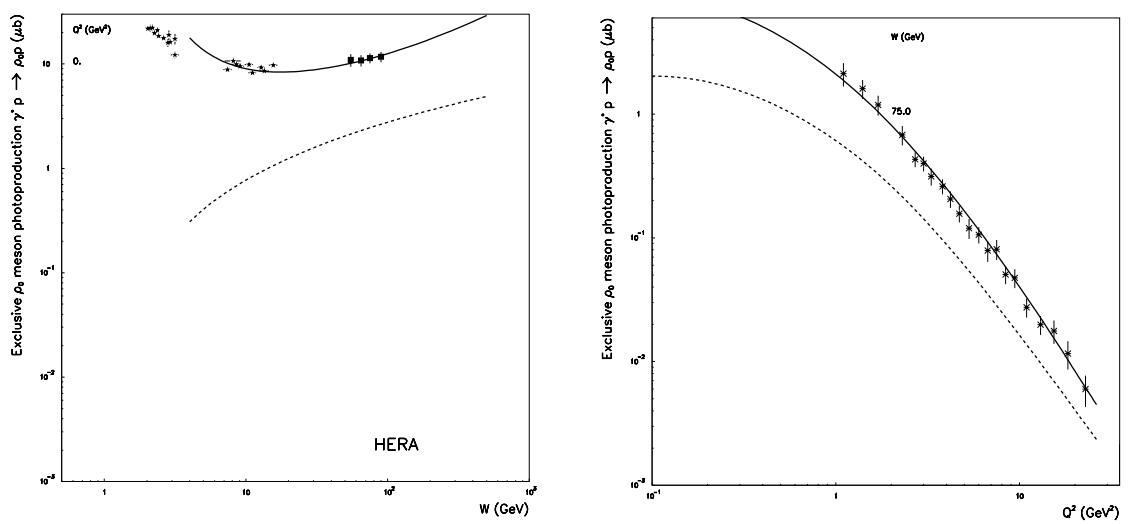

Figure 2. $\rho_{0}$ meson exclusive photoproduction cross-section (solid line)Eq. 32 in comparison with its asymptotic behaviour (dashed line) Eq. 33

\section{Conclusions}

As is seen from Fig.1 and Fig.2, the exclusive vector meson production may be described in the framework of extended Regge-eikonal approach and one does not need 'hard' pomerons with a high intercept in order to do it. The rise of cross sections when $Q^{2}$ is high is a transitory phenomenon dye to effective delay of asymptotic behaviour which is observed for low $Q^{2} 11$ or 12 . The $Q^{2}$-dependence is also described fairly well.

\section{References}

1. S. Troshin, N. Tyurin, Yad. Fiz. 40, 1008 (1984).

2. A. A. Logunov A. N. Tavkhelidze, Nuovo Cimento 29, 380 (1963).

3. L. Van-Hove, Phys. Lett. B 24, 183 (1967).

4. R. C. Arnold, Phys. Rev. D 153, 1533 (1967).

5. Cheng H., Wu T. T., Phys. Rev. D 186, 1611 (1969). Cheng H., Wu T. T., Phys. Rev. Lett. 24, 1456 (1970).

6. M. Froissart, Phys. Rev. D 123, 1053 (1961)

A. Martin, Phys. Rev. D 129, 993 (1963). 
7. R. J. M. Covolan, P. Degrolard, M. Giffon, L. L. Jenkovszky, E. Predazzi, Z. Phys. C 58, 109 (1993)

8. J. Finkelshtein, H. M. Fried, K. Kang, C-I Tan, Phys. Lett. B 232, 257 (1989)

E. S. Martynov, ibid., 367

9. C. Adloff et al., H1 Collaboration submitted to Eur. Phys. J C, DESY 99-010, hep-ex/9902019

10. V. A. Petrov in "Frontiers In Strong Interactions" Edited by P. Chiappetta, M. Haguenauer, J. Trân Thanh Vân. p.139

11. P. Desgrolard, A. Lengyel, E. Martynov, Eur.Phys.J. C 7, 655 (1999)

12. V. Petrov, A. Prokudin, hep-ph/9706257, Yad.Fiz 9621 (1999)

13. N. M. Kroll, T. D. Lee, B. Zumino Phys. Rev. D 157, 1376 (1967)

14. V. A. Petrov, A. P. Samokhin Phys. Lett. B 237, 500 (1990) 


\section{Results of fitting without the odderon contribution}

Hollow dots are $p p$ data and full dots are $\bar{p} p$ data. The dotted $(p p)$ and solid $(\bar{p} p)$ curves correspond to the model without the odderon contribution. The shadowed area corresponds to the region available by the uncertainty in the fitting parameters.
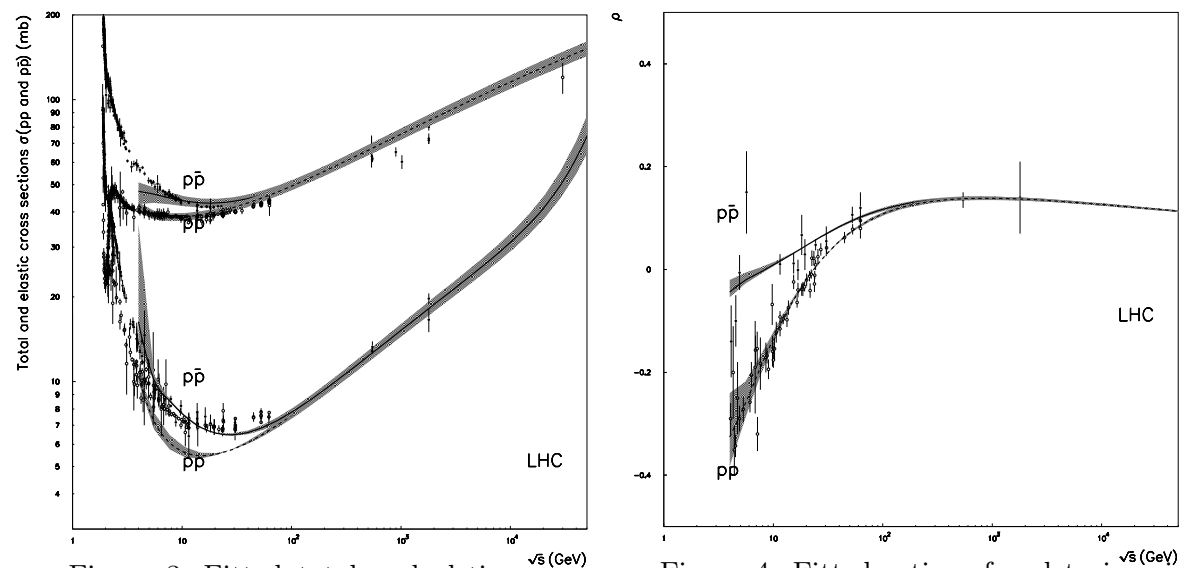

Figure 3. Fitted total and elstic cross $\operatorname{crec}^{\sqrt{s}(G \mathrm{eV})}$ tions. forward amplitudes.
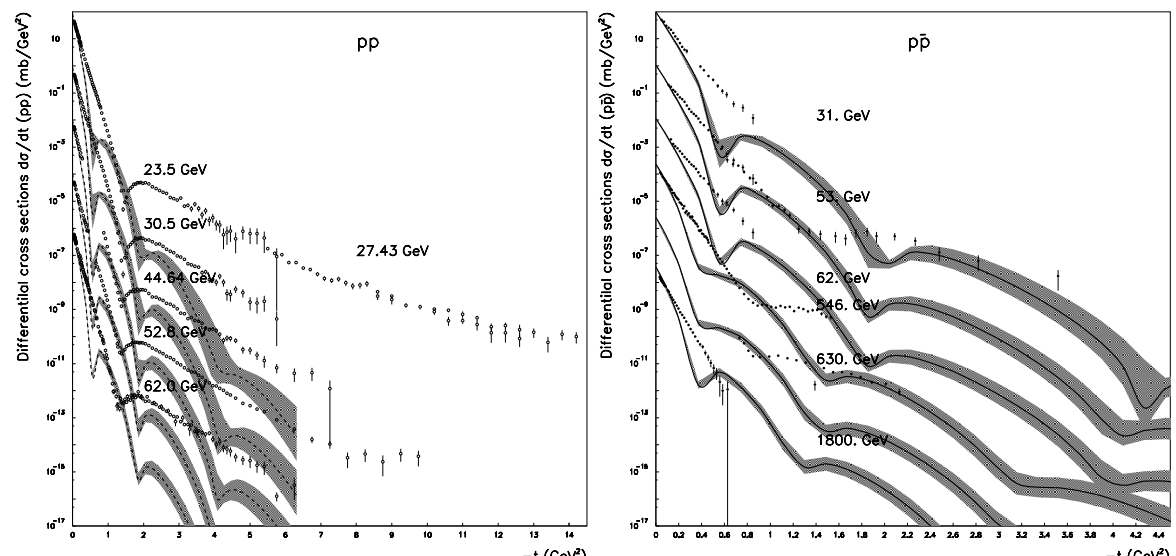

Figure 5. Fitted differential cross ${ }^{-t\left(\mathrm{Gev}^{2}\right)}$ sections Figure 6. Fitted differential cross ${ }^{-\mathrm{t}\left(\mathrm{Gev}^{2}\right)}$ sections $(p p)$. $(\bar{p} p)$. 


\section{Results of fitting with the odderon contribution}

Hollow dots are $p p$ data and full dots are $\bar{p} p$ data. The dotted $(p p)$ and solid $(\bar{p} p)$ curves correspond to the model with the odderon contribution. The shadowed area corresponds to the region available by the uncertainty in the fitting parameters.
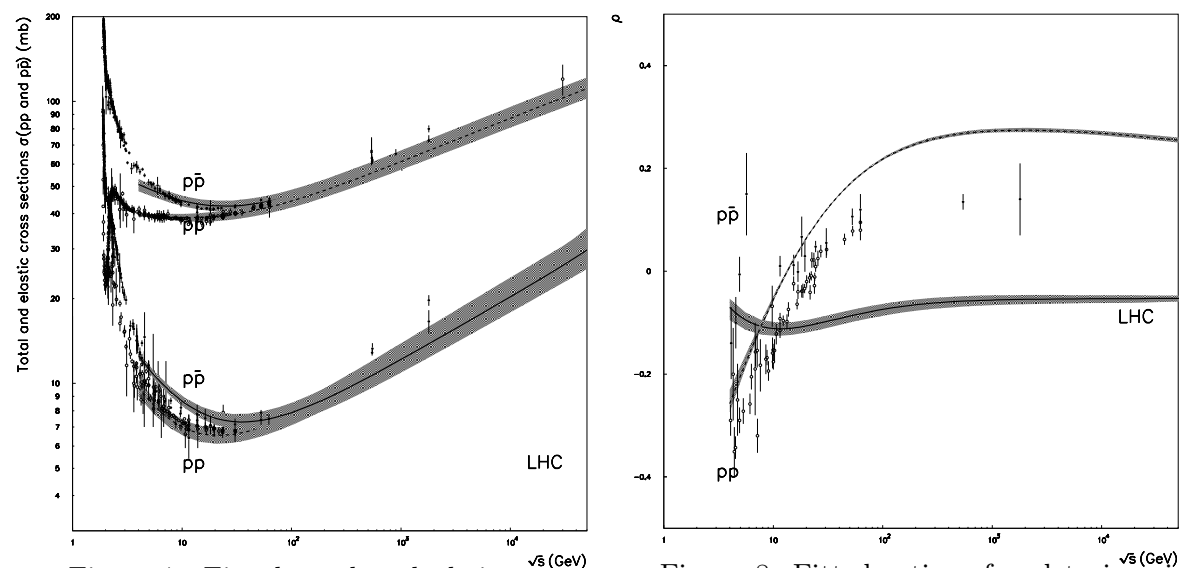

Figure 7. Fitted total and elstic cross $\mathrm{cec}^{\sqrt{\mathrm{s}}(\mathrm{GeV})}$ tions. forward amplitudes.
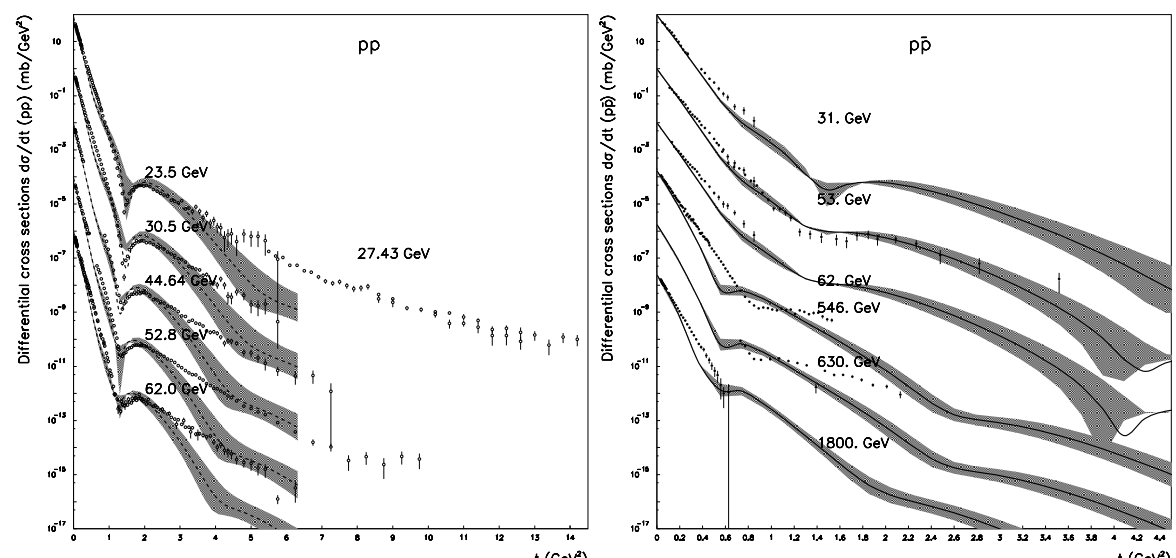

Figure 9. Fitted differential cross ${ }^{-t\left(\operatorname{cev}^{2}\right)}$ sections Figure 10. Fitted differential cross ${ }^{-t\left(\operatorname{cec}^{2}\right)}$ sections $(p p)$.

$(\bar{p} p)$. 